\title{
Quantitative Analysis of Peroxide Value in Virgin Coconut Oil by ATR- FTIR Spectroscopy
}

\author{
A.M. Marina*, W.I. Wan Rosli and M. Noorhidayah \\ School of Health Sciences, Health Campus, Universiti Sains Malaysia, 16150 Kubang Kerian, Kelantan, Malaysia
}

\begin{abstract}
Oxidation of fats and oils is an important indicator for performance and shelf life of oils. As other vegetable oils, virgin coconut oil (VCO) is not susceptible to lipid oxidation. Thus, a rapid method for quantitative analysis of peroxide value in VCO was developed in this study using Fourier transform infrared (FTIR) spectroscopy coupled with attenuated total reflectance (ATR). Thirty training samples of VCO were subjected to oxidative condition in convection oven at temperature of $70^{\circ} \mathrm{C}$ up to 20 days to accelerate the lipid oxidation process. The peroxide values were determined periodically using the standard method of American Oil Chemists' Society (AOCS) and then analyzed with FTIR spectroscopy. Calibration model using partial least square (PLS) was developed by studying the spectral features for regions that correlated with peroxide value content (absorption of oxidation compounds band near 988 to $900 \mathrm{~cm}^{-1}$ ) and validated by removing one sample at a time. A linear calibration curve was obtained for the actual value against FTIR predicted value which yields an equation of $y=1.0398 x+0.0468$ and satisfactory results of root mean square error of prediction (RMSEP) of 0.4978 and correlation coefficient $\left(\mathrm{R}^{2}\right)$ of 0.9826 . Thus, FTIR spectroscopy with PLS regression can serve as important tools for determining the peroxide value in VCO. Application of FTIR is rapid, accurate and environmental friendly compared to the conventional chemical analyses.
\end{abstract}

Keywords: FTIR, oxidation. peroxide value. virgin coconut oil.

\section{INTRODUCTION}

Virgin coconut oil (VCO) is considered to be one of the functional food oil due to its valuable therapeutic value [1]. Thus, its production is increasing especially in tropical countries. Though the increased in the production quantity shows a positive sign, it is important to ensure that the quality of the oil is preserve in order to obtain the full benefits from the oil.

The peroxide value is a measure of the concentration of peroxides and hydroperoxides forms in the initial stage of lipid oxidation. The number of peroxides present in vegetable oils reflects its oxidative level and thus its tendency to become rancid [2]. In addition, VCO is normally packed in transparent plastic bottles. During prolonged storage or display at retailers, VCO may undergo quality deterioration. Thus peroxide value is a very important quality parameter in $\mathrm{VCO}$ analysis and efficient method to measure the peroxide value content in VCO is called for.

The official wet chemistry method used to measure peroxide value in virgin coconut oil is the standard method of American Oil Chemists' Society. However, the method is time consuming and labour intensive despite being relatively simple. The use of infrared spectroscopy has been suggested as alternative method to determine the peroxide value in vegetable oils. Fourier transforms infrared spectroscopy

*Address correspondence to this author at the School of Health Sciences, Health Campus, Universiti Sains Malaysia, 16150 Kubang Kerian, Kelantan, Malaysia; Tel: 609-7677617; Fax: 609-7677515;

E-mail: mareena@kk.usm.my which employs the mid infrared region of electromagnet spectrum from 4000 to $400 \mathrm{~cm}^{-1}$. The ease feasibility of using FTIR is due to rapid technique ( 2 minutes) and reduces the use of toxic solvents [3]. FTIR has been reported for use of quantification of various oil parameters including peroxide value. Peroxide value has been determined using FTIR in palm oil [4], canola oil [3]; sunflower, olive, rapeseed and safflower oil [5]. Thus, this paper focuses on the development method to determine the peroxide value in VCO using FTIR with attenuated total reflectance (ATR) in conjunction with partial least square (PLS) statistical analysis.

\section{MATERIALS ANS METHODS}

\section{Materials}

Virgin coconut oil was obtained from Kelantan Biotech Corporation. All chemicals and reagents used were of analytical grade.

\section{Chemical Analysis}

Thirty training samples of VCO were subjected to oxidative condition in convection oven at temperature of $70^{\circ} \mathrm{C}$ up to 20 days to accelerate the lipid oxidation process. The peroxide values were determined periodically using the standard method of American Oil Chemists' Society (AOCS, $\mathrm{Cd}$ 8-53) [6]. Analyses were carried out in triplicate.

\section{Spectra Collection}

The FTIR measurement was done using Bruker Tensor 27 spectrometer (BRUKER, Karlsruhe, Germany) equipped with flow through horizontal ATR accessory. Drops of oil 


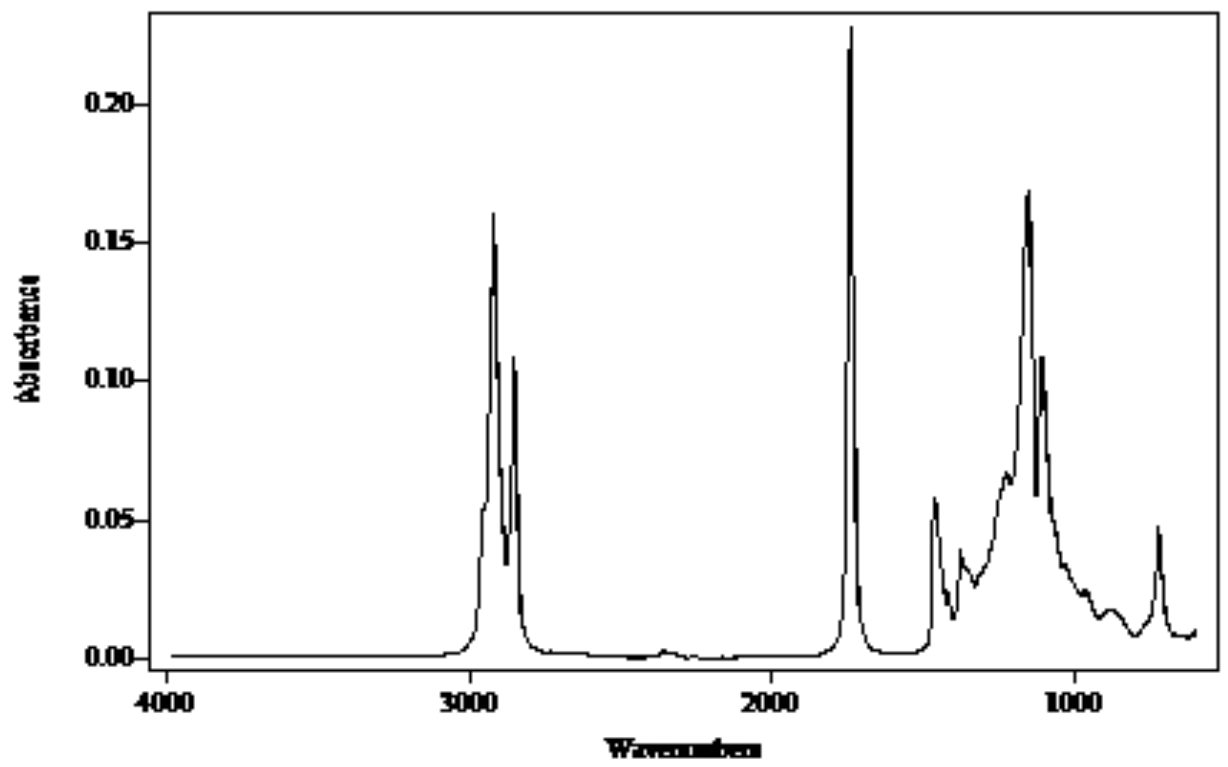

Fig. (1). FTIR spectrum of virgin coconut oil.

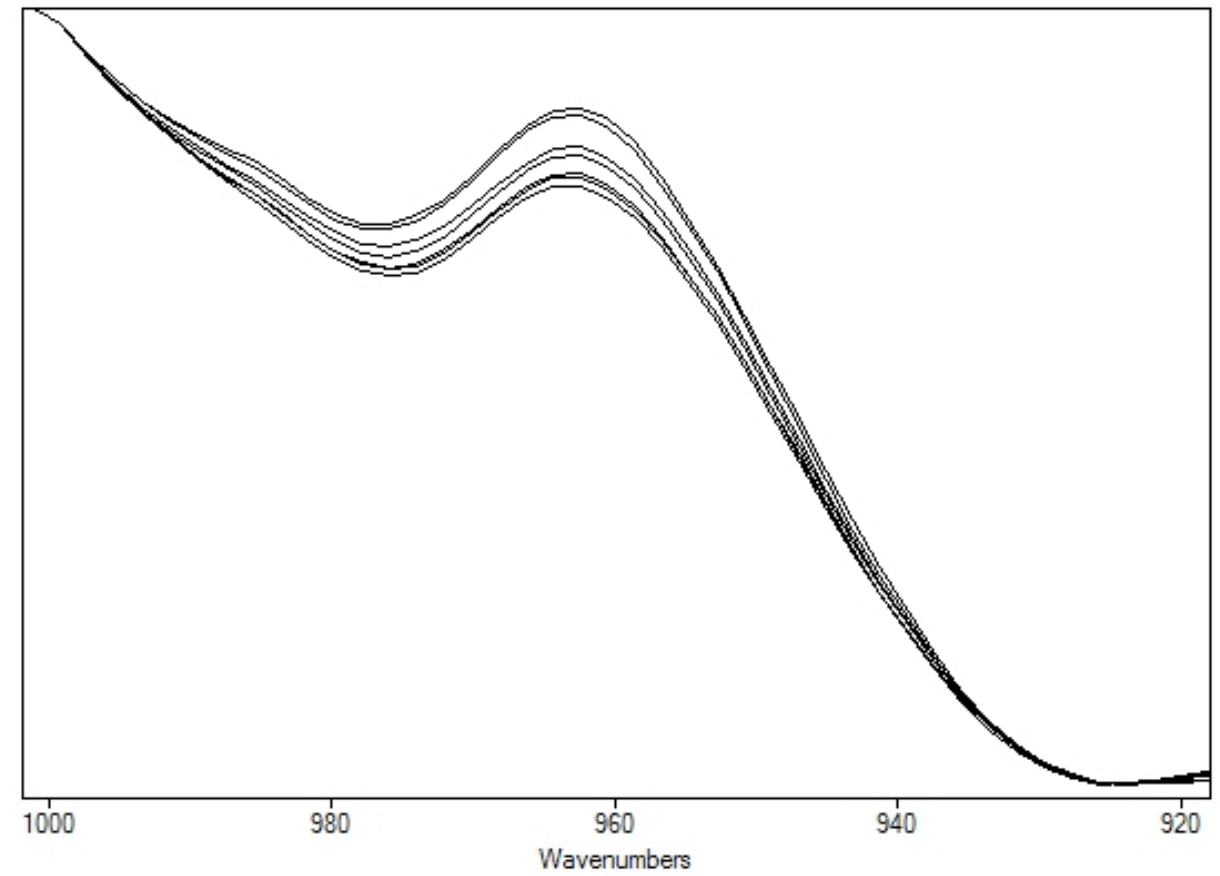

Fig. (2). Overlay spectra of virgin coconut oil at region 1000 to $920 \mathrm{~cm}^{-1}$.

samples were placed in contact with the ATR. Single beam spectra in the range of 4000 to $650 \mathrm{~cm}^{-1}$ were obtained and corrected against the background to present spectra in absorbance unit. Spectra were obtained using 32 scans at $4 \mathrm{~cm}$ resolution. Opus software (BRUKER, Karlsruhe, Germany) version 5.5 was used to operate the FTIR spectrometer and collect the spectra. The partial least square (PLS) calibration models that correlate between actual and predicted values of peroxide value was performed. The 'leave one out' cross validation was used to access the predictive accuracy of the calibration model.

\section{RESULTS AND DISCUSSION}

Fig. (1) shows the FTIR spectrum of VCO. One of the main feature of the spectrum is in the region of 3050 to 2800 $\mathrm{cm}^{-1}$ which was attributed to the absorbance of $\mathrm{CH}$ stretching vibrations (cis- $\mathrm{CH}=\mathrm{CH}-,-\mathrm{CH}_{2-}, \mathrm{CH}_{3}, \mathrm{CH}_{2} / \mathrm{CH}_{3}$ ) [4]. The region between 1400 to $900 \mathrm{~cm}^{-1}$ is due to the stretching of $\mathrm{C}-\mathrm{O}-\mathrm{C}$ and $\mathrm{CH}$ bending [7]. The $-\mathrm{C}=\mathrm{O}$ stretching absorption of the triacylglycerol ester linkage can be observed in the region from 1800 to $1650 \mathrm{~cm}^{-1}$. The region of 1500 to 1000 $\mathrm{cm}^{-1}$ is known as fingerprint region. The carbonyl absorption of the triacylglyceol linkage is observable at 1744 to 1739 $\mathrm{cm}^{-1}$ [8]. A band near $1238 \mathrm{~cm}^{-1}$ and $1163 \mathrm{~cm}^{-1}$ are related to the proportion of saturated acyl groups [9].

During thermal oxidation of VCO, several changes in the peak intensity were observed. The frequency for band 983 to $985 \mathrm{~cm}^{-1}$ were increased as the oxidation increased, reaching a maximum value near $988 \mathrm{~cm}^{-1}$ (Fig. 2). The band at 988 $\mathrm{cm}^{-1}$ is associated with presence of trans, trans and/or cism 


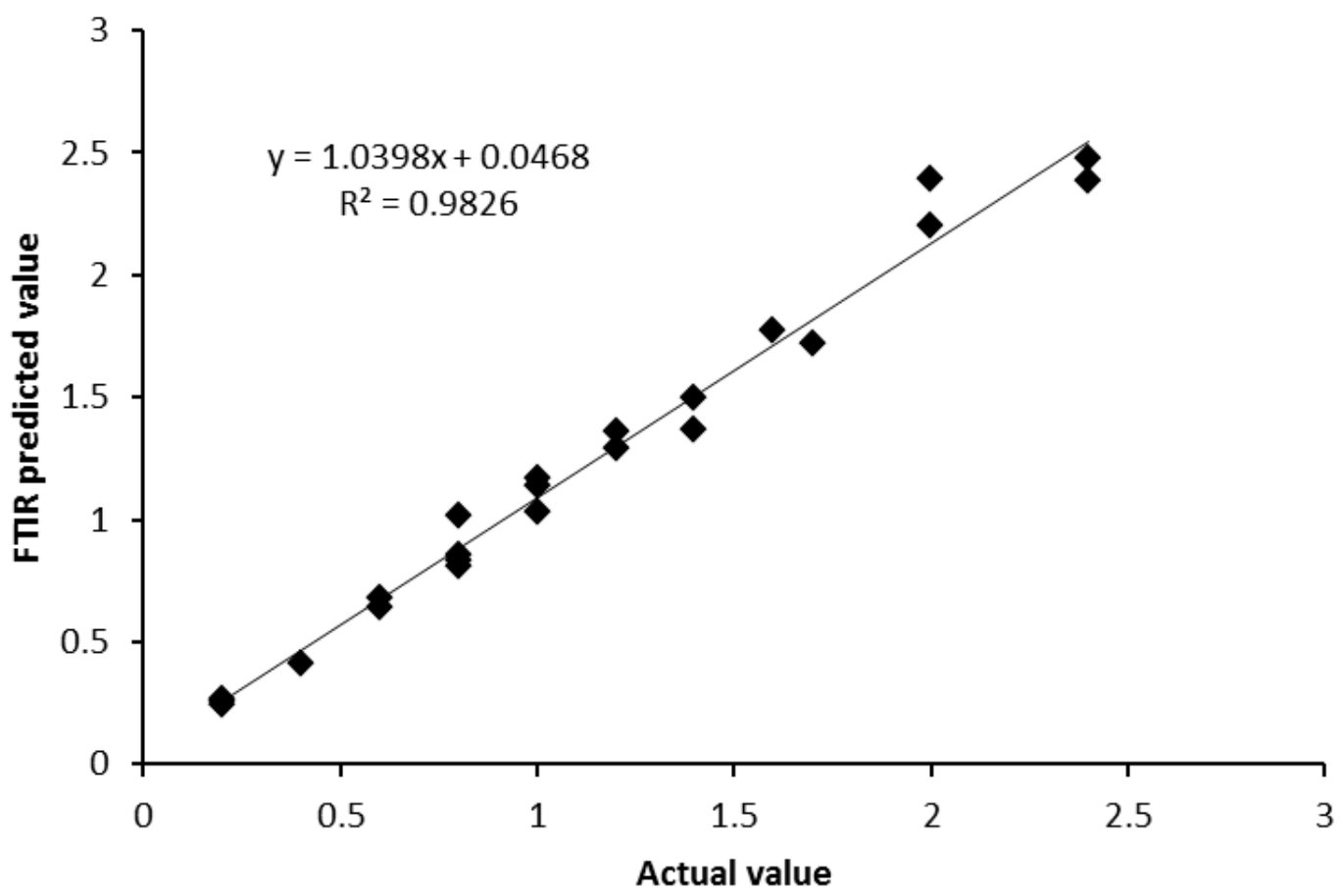

Fig. (3). PLS calibration model of actual versus predicted FTIR peroxide value.

trans-conjugated olefinic double bonds [10]. The band at 967 $\mathrm{cm}^{-1}$ is associated with the bending vibration of $\mathrm{CH}$ functional groups of trans-olefins [11]. Fig. (2) shows the increase in band $967 \mathrm{~cm}^{-1}$ as the oxidation advances. This band provided information about the gradual cis to trans isomerisation in the oxidize oil [12]. Thus, this band has been considered as indicator of oxidative stability.

The hydroperoxide bands have been reported at $3450 \mathrm{~cm}^{-}$ ${ }^{1}$, due to $-\mathrm{OO}-\mathrm{H}$ stretching vibration of the oxidized methyl octadecadieonate [4]. The formation on hydroperoxide band however was not observable in this study. This hydroperoxide band can be interfered by other $\mathrm{OH}$ containing components such as free fatty acids [3].

The ability of PLS to mathematically correlate spectral changes to changes in the concentration of component of interest is being utilize in this study. The region of 988-900 $\mathrm{cm}^{-1}$ was selected for PLS calibration. Fig. (3) shows the PLS calibration model of the actual peroxide value determined by chemical method versus the FTIR predicted value in VCO. The slope indicates that there is linear relationship between FTIR predicted and chemically determined values with slope approaching to 1 . The regression lines equation obtained was $y=1.0398 x+0.0468$ with coefficient of determination $\left(\mathrm{R}^{2}\right)$ equals to 0.9826 . Validation was done by removing one standard at a time and satisfactory result of root mean error of prediction (RMSEP) of 0.4978 was obtained.

\section{CONCLUSIONS}

The present study shows that reliable prediction ability is obtained for determination of peroxide value using PLS. This indicates that FTIR combines with PLS can serves as rapid powerful technique to determine the peroxide value in VCO than can be achieved using conventional methods.

\section{CONFLICT OF INTEREST}

The authors confirm that this article content has no conflicts of interest.

\section{ACKNOWLEDGEMENTS}

This research was financed by the Universiti Sains Malaysia short term grant (304/PPSK/61311055).

\section{REFERENCES}

[1] Marina, A.M.; Che Man, Y.B; Amin, I. Virgin coconut oil: Functional food oil. Trend Food Sci. Technol., 2009, 20, 481-487.

[2] Marina, A.M.; Che Man, Y.B.; Nazimah, S.A.H.; Amin, I. Chemical properties of virgin coconut oil. J. Am Oil. Chem Soc., 2009, 86, 301-307.

[3] Van de Voort, F.R.; Ismail, J.S.; Dubois, J.; Nicodemo, T. The determination of peroxide value by fourier transform infrared spectroscopy. J.Am Oil. Chem Soc., 1994, 71, 921-926.

[4] Moh, M.H.; Tang, T.S.; Che Man, Y.B.; Lai, O.M. Rapid determination of peroxide value in crude palm oil products using fourier transform infrared spectroscopy. J. Food Lipids, 1999, 6, 261-270.

[5] Guillen, M.D.; Cabo, N. Fourier transform infrared data versus peroxide and anisidine values to determine oxidative stability of edible oils. Food Chem., 2002, 77, 503-510.

[6] AOCS. Official Methods and Recommended Practice of the American Oil Chemists' Society, vol 5. AOCS Press: Champaign, 1996.

[7] Marina A.M.; Che Man, Y.B.; Nazimah, S.A.H.; Amin, I.; Syahariza, Z.A. Analysis of adulteration of virgin coconut oil by palm kernel olein using fourier transform infrared spectroscopy. $J$. Food Lipids, 2007, 14, 111-121.

[8] Guillen, M.D.; Cabo, N. Characterization of edible oils and lard by fourier transform infrared spectroscopy. Relationship between composition and frequency of concrete bands in the fingerprint region. J. Am Oil. Chem Soc., 1997, 74, 1281- 1286.

[9] Guillen, M.D., Cabo, N. Usefulness of the frequency data of the fourier transform infrared spectra to evaluate the degree of oxidation of edible oils. J. Agric. Food Chem., 1999, 47, 709-719. 
[10] Guillen, M.D.; Cabo, N. Some of the most significant changes in the fourier transform infrared spectra of edible oils under oxidative conditions. J. Sci. Food. Agric., 2000, 80, 2028-2036.

[11] Guillen, M.D.; Cabo, N. Study of the effects of smoke flavourings on the oxidative stability of the lipids of pork adipose tissue by means of fourier transform infrared spectroscopy. Meat Sci., 2004, 66, 646-657.

[12] Beltran, A.; Ramos, M.; Grane, N.; Martinm, M.L.; Garrigos, M.C. Monitoring the oxidation of almond oil by HS-SPME-GC-MS and ATR-FTIR. Application of volatile compounds determination to cultivar authenticity. Food Chem., 2011, 126, 603-609.

Received: May 29, 2013

Revised: August 14, 2013

Accepted: September 04, 2013

(C) Marina et al.; Licensee Bentham Open.

This is an open access article licensed under the terms of the Creative Commons Attribution Non-Commercial License (http://creativecommons.org/licenses/by$\mathrm{nc} / 3.0 /$ ), which permits unrestricted, non-commercial use, distribution and reproduction in any medium, provided the work is properly cited. 